\title{
An efficient AES and RC6 based cloud-user data security with attack detection mechanism
}

\author{
Shristi Bhute $^{1 *}$ and Siddhartha Kumar Arjaria ${ }^{2}$ \\ M.Tech Scholar, Department of Information Technology, TIT, Bhopal ${ }^{1}$ \\ Assistant Professor, Department of Information Technology, TIT, Bhopal ${ }^{2}$
}

\section{(C)2016 ACCENTS}

\begin{abstract}
This paper proposed an efficient framework for text data security. In this framework the data is totally controlled by the user and the user security is controlled by the cloud server. For data security advanced encryption standard (AES) and RC6 algorithms are used. The data is uploaded by the users so the data of the concern user is the admin of those data. Cloud servers are also not allowed to view or update the data without the permission of the concern user. When any user or cloud server wants to access the data of others, the data can be requested. The encrypted data is send after the permission of the concern user. It can be decrypted by AES and RC6 key. Bit rendering and notification can be helpful in malicious behavior detection. The results shows that our approach is better in terms of using the private key, key randomization and provides the support of user to user, user to cloud server and cloud server to user as comparison to the traditional approach.
\end{abstract}

Keywords

Cloud computing, Data security, AES, RC6.

\section{Introduction}

There are a few causes why business undertakings might need to receive the help of cloud computing. Cloud computing give ease in administration. The two primary parts of cloud computing are base and programming applications. The principal underpins the equipment assets like server, stockpiling and system parts. The second backings the product applications and figuring power for running business applications, as gave by the outsiders. Circulated figuring give on interest assets in light of pool of advantages accessible by the cloud suppliers [1-3]. From the bit of standard enrolling the upsides of scattered enlisting are: dexterity, lower section cost, gadget independency, zone independency, and flexibility [4, 5]. Regardless, the security concerns are the bona fide key viewpoints later on coursed preparing time. There are a couple security majors are shown in [5-10].Virtualization, overwhelming enlisting are additionally the more prominent office parts of passed on handling. Notwithstanding, to satisfy the execution on the parallel framework and keeping up the respectability is convincing [11].

\footnotetext{
*Author for correspondence
}

In every one of these works, amazing tries are made to game plan approaches that meet different essentials: high course of action practicality, stateless check, unbounded use of request and wretchedness of information, and so forth. Considering the part of the verifier in the model, every one of the arrangements showed before fall into two classes: private auditability and open auditability [5]. Despite the way that orchestrates with private auditability can play out the arrangements sufficiently, yet it is attempting circumstance if the information is securing unobtrusively [5]. Virtualization is the key fragments of scattered figuring by which information sharing is conceivable between various machines of virtual proximity from the server develop [12]. Virtualization connects with the live movement [9] of virtual machines (i.e. moving a VM starting with one host then onto the accompanying without chopping it down) which helps in keeping up the guaranteed SLA to the cloud client other than to alter stack crosswise over physical servers in the information centres[12]. Security aspect is more crucial in today's aspect [13].

The essential cloud suppliers are Google, Microsoft, Amazon and Salesforce.com. The dispersed figuring advantage show relies on upon the data correspondence layer. The whole correspondence is 
relies on upon three layers. The essential layer is Software as a Service (SaaS) which is generally changed on desktop based applications into internet programming things that can be used far and wide. A generally utilized application is Salesforce.com, a client relationship association (CRM) programming for interfacing with affiliations and customers [14]. As appeared by [14] Platform as a Service (PaaS) is a condition for Cloud Computing Security Management for making and building applications for various circumstances. As appeared by Infrastructure as a Service (IaaS) for the most part fuses virtualization circumstances as got associations rather than physical or submitted PC hardware.

In the standard method for figuring the advantages are picked up locally which are once in a while higher in expense and not sensible. This obliges the courses in which a client could take an interest with the thing in that the thing was just open and available for the primary workstation [14]. Regardless, now by the use of passed on figuring the Software as a Service show has changed this reasonability in a way that thing can be acquired for use over the Internet [14]. Instead of getting programming in a boxed course of action, the client can buy an association to utilize an application that is empowered in the cloud [14]. The standard great position of this sort of structure arrives is no need of genuine work station as the client run yet on interest assets/programming can offer it to lease [15]. So in the event that it is joined with the security associations it winds up being amazing [16]. Cloud data security is important from the both of the end [17]. This is the main objective of our research work.

\section{Literature survey}

In 2011, Wang et al. [18] recommended that conveyed figuring has been envisioned as the bleeding edge fabricating plan of IT Enterprise. It moves the application programming and databases to the thought broad server cultivates, where the organization of the data and organizations may not be totally reliable. A maker focuses on the issue of ensuring the respectability of data stockpiling in Cloud Computing. In particular, they consider the task of allowing a pariah controller (TPA), for the advantage of the cloud client, to check the dependability of the dynamic data set away in the cloud. The presentation of TPA slaughters the relationship of the client through the assessing of whether his data set away in the cloud is without a doubt set up, which can be fundamental in finishing economies of scale for Cloud Computing.
In 2012, Naqvi et al. [19] exhibit a formal technique for testing the impact of versatility and heterogeneity on the assembled Cloud security organizations. Their hopes to develop a mean of measuring the impact on security limits under various working conditions and parameters of brought together Cloud courses of action. Their eventual outcomes of this work will help associations with recognizing the best security basic arranging that will fit their Cloud designs and execution requirements.

In 2012, Tianfield et al. [20] introduce a thorough study on the troubles and issues of security in dispersed figuring. They first explore the impacts of the unmistakable traits of disseminated figuring, to be particular, multi-residency, adaptability and pariah control, upon the security requirements. By then, they analyze the cloud security necessities in regards to the primary issues, i.e., protection, respectability, availability, trust, and survey and consistence. They discuss the exploratory order for security issues in disseminated figuring.

In 2012, Abuhussein et al. [21] suggest Healthcare, preparing, business, and various diverse territories look at circulated processing as an attempt to understand the constant inadequacy in volume, establishment, accessibility, and watching quality. Then again, moving data to the cloud proposes moving control of the customer's data to the cloud organization supplier uncertainly.

In 2012, Liu et al. [22] recommend that the security issue of appropriated registering is essential and it can keep the quick change of circulated figuring. It exhibits some appropriated processing structures and separates conveyed figuring security issue and its strategy as demonstrated by the disseminated registering thoughts and characters. The data insurance and organization openness in circulated processing are the key security issue. Single security procedure can't handle the appropriated figuring security issue and various customary and new advances and techniques should be used together to ensure the total disseminated processing structure.

In 2013, Pant et al. [23] exhibit the methodology for cloud allotment and cloud security examination to explore potential security and consistence proposals in cloud environment. They discusses in inconspicuous component on how an affiliation may proceed for security and consistence examination in the midst of the cloud estimation. Their strategy and thoughts point by point in this paper would be 
important for affiliations that are incorporated into the cloud gathering prepare.

In 2013, Du meng et al. [24] discusses dispersed registering data security issues, including tile security of data transmission, stockpiling, security and organization of security. Focus on comprehensive data organization impact cloud security examination, and called attention to that a jump forward in the headway of this dispersed processing, endeavor to list the looking at philosophies and whole deal change heading.

In 2013, Yang et al. [25] prescribed that the data security and assurance on cloud is a basic issue, transforming into the best obstruction of conveyed processing headway. A Trusted Cloud Computing Platform (TCCP) in perspective of remote approval builds a trusted cloud for tenant.

In 2014, Zhao et al. [26] proposed homomorphism encryption calculation in the distributed computing to take care of the issue of information security. As per the creators this can fit for handling and recovery of the encoded information and it is viably pertinent for information transmission and the storage.

In 2015, Gupta et al. [27] has been imagined as a front line basic arranging of IT Industries. Security and insurance is the noteworthy snag in the cloud environment as a consequence of its straightforward development displaying. They researches the cloud security risks moreover discusses the ebb and flow security approaches to manage secure the cloud environment .They also proposed a novel Triframework for cloud security against data break which give all around security to the cloud basic arranging.

In 2015, Liu et al. [28] suggest that the existing privacy-preserving rule mining methods only assume a distributed model where every data owner holds the self-data without encryption and together follow a secure protocol to perform rule mining. To address this limitation, they have proposed a novel Protocol for Outsourced Rule Mining (PORM) in this paper. PORM performs rule mining in a cloud environment where data are both encrypted and outsourced.

\section{Proposed algorithm}

In this approach a secure circular cloud-user framework has been proposed. For maintaining data security AES and RC6 mechanism have been applied for data security. In this approach the data can be 112 uploaded on the any one of the available server from the total three servers. One server is used for backup and data pre-processing by the cloud server. So there are total of four servers. Three servers for data uploading and one server are dedicated for the cloud server.

Data virtualization is maintained in this framework according to the size allocated by the hard drive. It will automatically update according to the need and use by the cloud user. There are three different cases can be handled easily which are as under through our cloud computing framework:

- User-User Security: In this mode of data security the textual data can be uploaded from any registered user in the cloud environment. This data is open for the users which have uploaded this data but automatically upon the data uploading AES and RC6 algorithms are applied to these data. So other users can request the file but cannot view it directly. After the approval of the source admin it can be seen in the client side. But the data is in the form of encryption file. After applying two encryption keys the data can be shown permanently to the concern user.

- Cloud-User Security: This mode supports the text data uploading from any user to the cloud server. This text data is secured by applying AES and RC6 algorithms.

- User-Cloud Security: This mode supports the security of the user data from the cloud server. This user data is also secured from AES and RC6 algorithms. So the cloud server have not permission to check the data and see it directly.

The data is exchanged with AES and RC6component framework and the plaintext is changed to content as demonstrated by AES and RC6 both. It gives two key securities. By then a data bit archive is send with the data that will thusly render the notification to the organization supplier if the not relegated customer will open the record first. As the security is by standard encryption system it will give a prevalent and strong against dissent of administration. This is the third thought incorporated our framework. By then the recipient can get to the data resulting to applying both AES and RC6 encryption standard segment. If some other customer opens the records the data bit cautions the mishandle operation to the cloud supplier. The keys are sporadic made so for the same record the keys are particular. So tailing it is particular. The whole mechanism can be easily understandable by Figure 1 . 


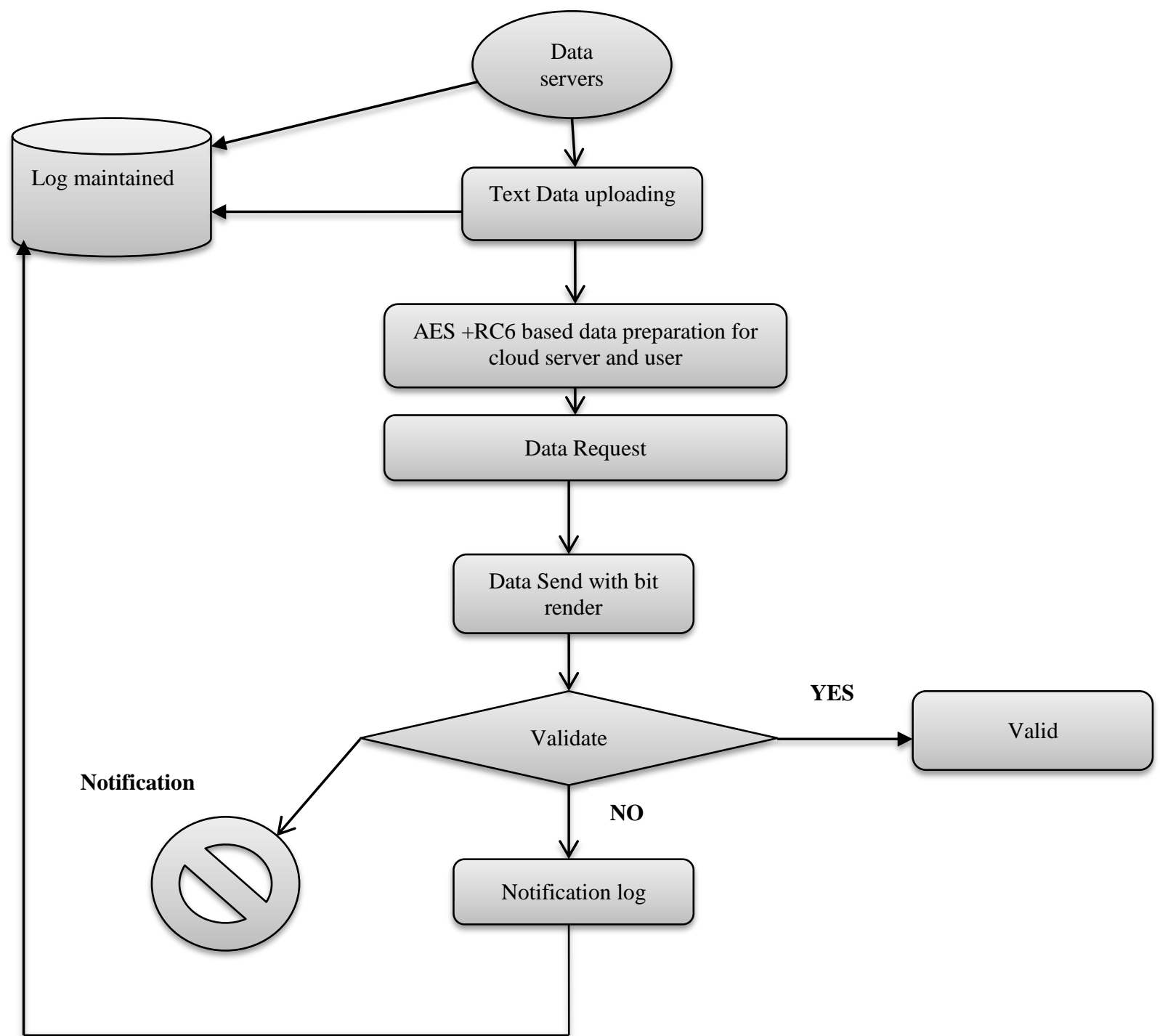

Figure 1 Work flow

\section{Algorithm 1: AES Algorithm}

128-bit key has been used in AES algorithm [29].

Step 1: Plain text content as an information.

Step 2: The key that is given as information is wandered into a group of 44 words (32-bits each), w[i]. 4 particular words (128 bits) serve as a round key for each round.

Step 3: 4 particular stages are used, 1 change and 3 of substitution:

- Substitute bytes - Uses a S-box to play out a byteto-byte substitution of the piece

- Shift - An essential change

- Mix segments - A substitution that makes usage of number juggling.

- Add round key - A direct bitwise XOR of the present square with the bit of the degree.
Step 4: It demonstrates the encryption round utilizations number-crunching in the limited field that is Galois field GF (27), with the irreducible polynomial.

Step 5: Just the Add Round Key stage uses the key. Whatever other stage is reversible without learning of the key.

Step 6: The Add Round Key is a sort of Vernam figure and free from any other individual would not force. The other 3 arranges together give confusion, scattering, and nonlinearity, however without any other person would give no security in light of the way that they don't use the key. At that point the information is modifying as indicated by the XOR encryption with the additional round key. The stage is additionally totally reversible. 


\section{Algorithm 2: RC6 Algorithm [30]}

Step 1: b byte preloaded into the c-word

Step 2: L $[0, \ldots, c$ - 1]

Step 3: Number $r$ of rounds [all the initialization are random]

$\mathrm{x}=$ First initialization

$\mathrm{y}=$ Second initialization

Yield:

Step 4: w-bit round keys $\mathrm{S}[0, \ldots, 2 \mathrm{r}+3]$

Strategy:

$\mathrm{S}[0]=\mathrm{X}$

Step 5: for $\mathrm{i}=1$ to $(2 \mathrm{r}+3)$ do

$\mathrm{S}[\mathrm{i}]=\mathrm{S}[\mathrm{i}-1]+\mathrm{y}$

Step 6: Each block key is generated until reached to the end of file vector.

Step 7: Shifting process

$\mathrm{A}=\mathrm{S}[\mathrm{i}]=(\mathrm{S}[\mathrm{i}]+\mathrm{A}+\mathrm{B})<<<3$

Step 8: Then again shifting is performed with the 3 bit java shifting to make the substitution matrix.

Step 9: The whole process is applied to the whole block division achieved.

Step 10: The final key is generated according to the $r$ rounds.

Step 7: End;

\section{Results and evaluation}

Diverse tables are utilized to keep up productively the information in the cloud environment at the server side in our methodology. The notification times demonstrates this component with time estimation when server knows the data about the change of information. Our cloud server notification time demonstrates that our approach provides the rendering in the very short duration. The below tables and figures demonstrated our results achieved. Table 1 shows the data information along with the RC6 and AES keys. Table 2 shows the collective time in the process of encryption and decryption. Table 3 shows the bit rendering time along with the notification time to the cloud server. Figure 2 and Figure 3 demonstrated the time taken by the process in the encryption and decryption during the encryption process of Rc6 and AES. Figure 4 shows the results of rendering and notification. Table 4 shows the comparison between the traditional approach [28] and the proposed approach. It shows that our approach is better in terms of using the private key, key randomization and provides the support of user to user, user to cloud server and cloud server to user. The key size variability is also an advancement of this approach.

Table 1 Data keys and other information

\begin{tabular}{lllllll}
\hline File name & User name & Server name & Upload date & Open status & RC6 key & AES key \\
\hline c3.txt & user123456 & server4 & Thu Jul 14 21:57:42 IST 2016 & no & mV9IcB5Qh4 & 174e9 \\
c5.txt & user12345 & server4 & Sat Jul 16 16:53:42 IST 2016 & no & tU6CrH2Qi2 & 17768 \\
eee.txt & amit2 & server4 & Sun Jul 03 19:40:41 IST 2016 & yes & tL5BfF5Bf6 & 17d50 \\
c1.txt & user123456 & server4 & Thu Jul 14 21:57:30 IST 2016 & yes & jI4FpB5Ub4 & $17 \mathrm{e} 47$ \\
c4.txt & user123456 & server4 & Sat Jul 16 16:43:28 IST 2016 & no & rM0AqD5Cb6 fffe86aa \\
ff.txt & amit2 & server4 & Sun Jul 03 19:43:31 IST 2016 & no & rS7LdD1Fh6 & fffe8e18 \\
\hline
\end{tabular}

Table 2 Encryption (E) and decryption (D) time in MS

\begin{tabular}{lllllll}
\hline File name & Status & E-RC6 & D-RC6 & E-AES & D-AES & Status \\
\hline c3.txt & attack & 20372 & 20094 & 193 & 0 & attack \\
c5.txt & attack & 31751 & 31155 & 401 & 0 & attack \\
eee.txt & safe & 5 & 5 & 25 & 3 & safe \\
c1.txt & safe & 7136 & 7008 & 233 & 247 & safe \\
c4.txt & attack & 27514 & 27102 & 472 & 0 & attack \\
ff.txt & attack & 1252 & 1244 & 32 & 0 & attack \\
\hline
\end{tabular}

Table 3 Bit rendering status

\begin{tabular}{lllll}
\hline User name & File name & Rendering time & Notification time & Margin in MS \\
\hline amit2 & ff.txt & Sun Jul 03 20:03:47 IST 2016 & Sun Jul 03 20:03:47 IST 2016 & 29 \\
user123456 & c3.txt & Thu Jul 14 22:23:28 IST 2016 & Thu Jul 14 22:23:28 IST 2016 & 86 \\
user123456 & c4.txt & Sat Jul 16 16:44:12 IST 2016 & Sat Jul 16 16:44:12 IST 2016 & 48 \\
user12345 & c5.txt & Sat Jul 16 16:53:54 IST 2016 & Sat Jul 16 16:53:54 IST 2016 & 75 \\
\hline
\end{tabular}




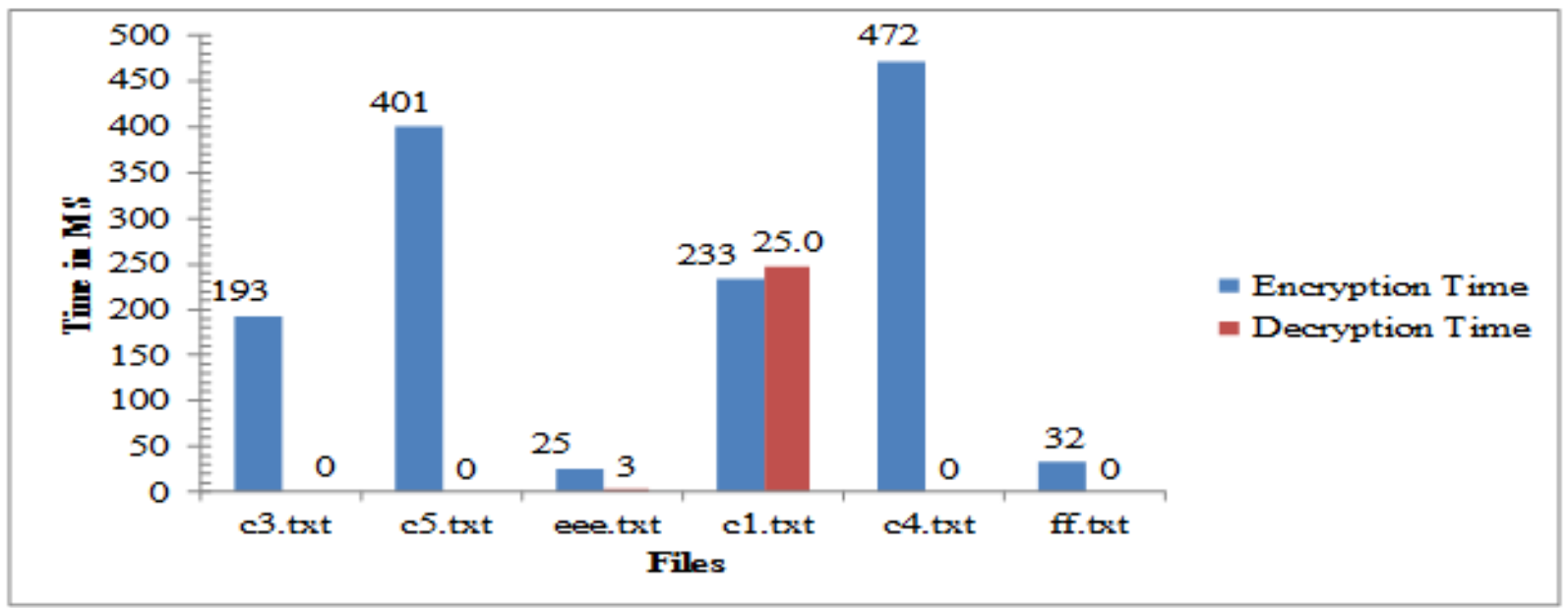

Figure 2 Encryption and decryption time comparison based on AES

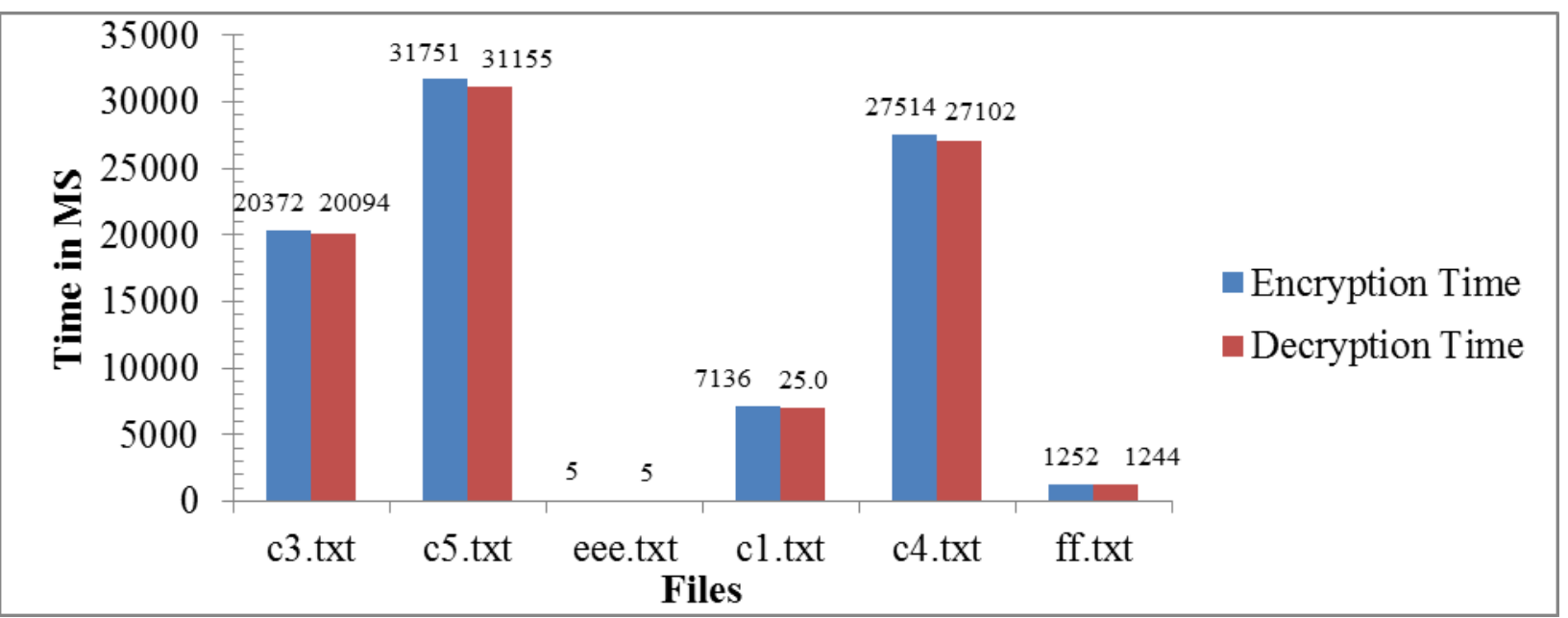

Figure 3 Encryption and decryption time comparison based on RC6 + AES

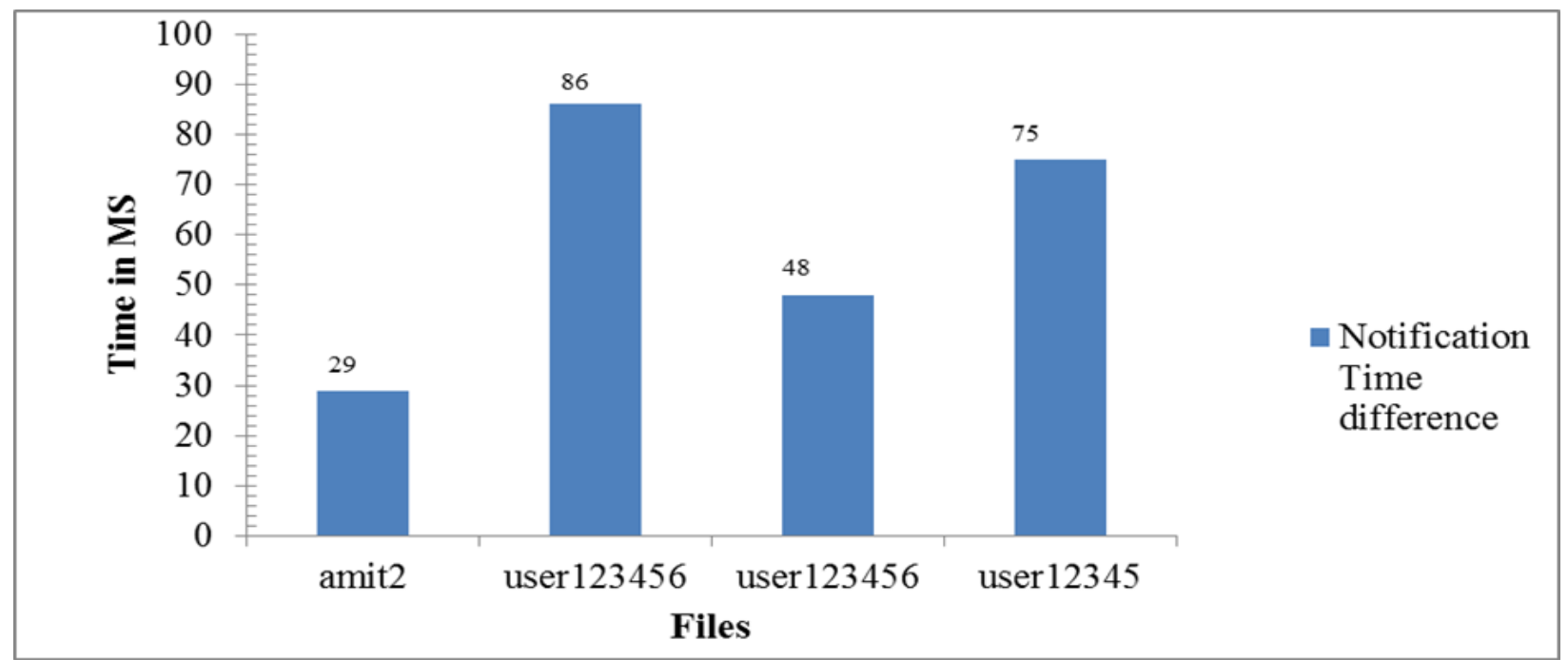

Figure 4 Attack analysis 
Shristi Bhute et al.

Table 4 Comparative study

\begin{tabular}{|c|c|c|c|}
\hline S. No & Attributes & {$[28]$} & Proposed work \\
\hline 1 & $\begin{array}{l}\text { Number of } \\
\text { Messages }\end{array}$ & 2 & 4 \\
\hline 2 & Total randomization & 1 & 2 \\
\hline 3 & $\begin{array}{l}\text { Total } \\
\text { Data } \\
\text { Encryption }\end{array}$ & 1 & 2 \\
\hline 4 & $\begin{array}{l}\text { Total } \\
\text { Data } \\
\text { Decryption }\end{array}$ & 1 & 2 \\
\hline 5 & Key Length variability(bits) & NA & $512-2048$ \\
\hline 6 & Property 1 (User) & Multi-user & Multi-user \\
\hline 7 & Property 2 (Security Property) & $\begin{array}{l}\text { User to user, User to cloud } \\
\text { server }\end{array}$ & $\begin{array}{l}\text { User to user, User to cloud server, Cloud server to } \\
\text { user }\end{array}$ \\
\hline 8 & Property 3 (Data Handling) & Frequent data & Frequent and non-frequent data \\
\hline 9 & Property 4 (Data Type) & Text & Text \\
\hline 10 & Property 5 (Symmetric Key) & One & One \\
\hline 11 & Property 6 (Private key) & One & Two \\
\hline 12 & Property 7 (Public key) & One & One \\
\hline
\end{tabular}

\section{Conclusion}

This paper demonstrated an efficient secure user cloud framework with the help of AES and RC6 algorithms. This approach provides the flexibility of inter clod communication with secure transactions by using AES and RC6 mechanism. Virtualization is being adopted through run time and maintains flexibility for the cloud users. The key control support mainly through the cloud user and the cloud user is controlled by the cloud server but the total control on the data is on the hand of cloud users. So that proper data security can be applied. Bit rendering and notification can be helpful in malicious behavior detection. The results shows that our approach is better in terms of using the private key, key randomization and provides the support of user to user, user to cloud server and cloud server to user as comparison to the traditional approach.

\section{Acknowledgment}

None.

\section{Conflicts of interest}

The authors have no conflicts of interest to declare.

\section{References}

[1] Armbrust M, Fox A, Griffith R, Joseph AD, Katz RH, Konwinski A, et al. Above the clouds: a Berkeley view of cloud computing. University of California, Berkeley, Technical Report No. UCB/EECS-2009-28.

[2] Ruiz-Agundez I, Penya YK, Bringas PG. Cloud computing services accounting. International Journal of Advanced Computer Research (IJACR). 2012; 2(4):1-17.

[3] Singh MA, Shrivastava M. Overview of security issues in cloud computing. International Journal of Advanced Computer Research.2012; 2(3):41-5.

[4] Ateniese G, Burns R, Curtmola R, Herring J, Kissner $\mathrm{L}$, Peterson $\mathrm{Z}$, et al. Provable data possession at untrusted stores. In proceedings of the 14th ACM conference on computer and communications security 2007 (pp. 598-609). ACM.

[5] Dubey AK, Dubey AK, Namdev M, Shrivastava SS. Cloud-user security based on RSA and MD5 algorithm for resource attestation and sharing in java environment. In sixth international conference on software engineering (CONSEG) CSI 2012 (pp. 1-8). IEEE.

[6] Juels A, Kaliski Jr BS. PORs: Proofs of retrievability for large files. In proceedings of the 14th ACM conference on computer and communications security 2007 (pp. 584-97). ACM.

[7] Shacham H, Waters B. Compact proofs of retrievability. In international conference on the theory and application of cryptology and information security 2008 (pp. 90-107). Springer Berlin Heidelberg.

[8] Alotaibi MB. Antecedents of software-as-a-service (SaaS) adoption: a structural equation model. International Journal of Advanced Computer Research. 2016; 6(25):114-29.

[9] Naor M, Rothblum GN. The complexity of online memory checking. In 46th annual IEEE symposium on foundations of computer science (FOCS) 2005 (pp. 573-82). IEEE.

[10] Tsai WT, Sun X, Balasooriya J. Service-oriented cloud computing architecture. In seventh international 
conference on information technology: new generations (ITNG) 2010 (pp. 684-9). IEEE.

[11] Patra GK, Chakraborty N. securing cloud infrastructure for high performance scientific computations using cryptographic techniques. International Journal of Advanced Computer Research. 2014; 4(4):66-72.

[12] Pachorkar N, Ingle R. Multi-dimensional affinity aware VM placement algorithm in cloud computing. International Journal of Advanced Computer Research. 2013; 3(4):121-5.

[13] Conteh NY, Schmick PJ. Cybersecurity: risks, vulnerabilities and countermeasures to prevent social engineering attacks. International Journal of Advanced Computer Research. 2016; 6(23):31-8.

[14] Tschinkel B. Cloud computing security understanding risk areas \& management techniques.

[15] Zheng L, Hu Y, Yang C. Design and research on private cloud computing architecture to support smart grid. In international conference on intelligent humanmachine systems and cybernetics (IHMSC) 2011 (pp. 159-61). IEEE.

[16] Li M, Yu S, Cao N, Lou W. Authorized private keyword search over encrypted data in cloud computing. In international conference on distributed computing systems (ICDCS) 2011(pp. 383-92). IEEE.

[17] Shristi B, Arjaria SK. Security of text data outsourcing in cloud computing. International Journal of Advanced Technology and Engineering Exploration. 2016; 3(20): 91-7.

[18] Wang Q, Wang C, Ren K, Lou W, Li J. Enabling public auditability and data dynamics for storage security in cloud computing. IEEE Transactions on Parallel and Distributed Systems. 2011; 22(5):847-59.

[19] Naqvi S, Michot A, Van de Borne M. Analysing impact of scalability and heterogeneity on the performance of federated cloud security. In IEEE 11th international conference on trust, security and privacy in computing and communications 2012 (pp. 113742). IEEE.

[20] Tianfield H. Security issues in cloud computing. In IEEE International conference on systems, man, and cybernetics (SMC) 2012 (pp. 1082-9). IEEE.
[21] Abuhussein A, Bedi H, Shiva S. Evaluating security and privacy in cloud computing services: a stakeholder's perspective. In international conference for internet technology and secured transactions 2012(pp. 388-95). IEEE.

[22] Liu W. Research on cloud computing security problem and strategy. In international conference on consumer electronics, communications and networks (CECNet) 2012 (pp. 1216-19). IEEE.

[23] Pant N, Parappa S. Seeding the cloud in a secured way: cloud adoption and security compliance assessment methodologies. In IEEE international conference on software engineering and service science (ICSESS) 2013 (pp. 305-8). IEEE.

[24] Meng D. Data security in cloud computing. In international conference on computer science \& education (ICCSE) 2013 (pp. 810-3). IEEE.

[25] Yang F, Pan L, Xiong M, Tang S. Establishment of security levels in trusted cloud computing platforms. In green computing and communications (GreenCom), IEEE and internet of things (iThings/CPSCom), IEEE international conference on and IEEE cyber, physical and social computing 2013 (pp. 2119-22). IEEE.

[26] Zhao F, Li C, Liu CF. A cloud computing security solution based on fully homomorphic encryption. In 16th international conference on advanced communication technology 2014 (pp. 485-8). IEEE.

[27] Gupta A, Chourey V. Cloud computing: security threats \& control strategy using tri-mechanism. In international conference on control, instrumentation, communication and computational technologies (ICCICCT) 2014 (pp. 309-16). IEEE.

[28] Liu F, Ng WK, Zhang W. Encrypted association rule mining for outsourced data mining. In IEEE international conference on advanced information networking and applications 2015 (pp. 550-7). IEEE.

[29] Singhai P, Shrivastava A. A review on Image Security through standard encryption techniques. International Journal of Advanced Technology and Engineering Exploration. 2015; 2(12):170-4.

[30] Rivest RL, Robshaw MJ, Sidney R, Yin YL. The RC6TM block cipher. In first advanced encryption standard (AES) conference 1998. 